Trauma Berufskrankh 2014 · 16[Suppl 3]:219-222 DOI 10.1007/s10039-014-2072-8

Online publiziert: 15. Mai 2014

๑) Springer-Verlag Berlin Heidelberg 2014

Th. Köhler

Deutsche Gesetzliche Unfallversicherung (DGUV), Landesverband Südwest, Heidelberg

\title{
Bericht zur
} sozialpolitischen Entwicklung

schen einem öffentlichen und einem gewerblichen UV-Träger (Unfallversicherungsträger) die Rechtsgrundlage gefehlt. Das ist eine weitere wichtige Etappe in der strukturellen Neuordnung der gesetzlichen Unfallversicherung.

Im öffentlichen Sektor ist das politische Ziel von einer Unfallkasse pro Bundesland noch nicht überall erreicht. So gibt es beispielsweise in Niedersachsen noch 5 UV-Träger der öffentlichen Hand.

Die Fusion im landwirtschaftlichen Bereich erfolgte über gesetzliche Regelungen. Seit 01.01.2013 gibt es die Sozialversicherung für Landwirtschaft, Forsten und Gartenbau (SVLFG) mit Sitz in Kassel; in ihr gingen alle vorher selbstständigen Organisationen, auch die landwirtschaftlichen Berufsgenossenschaften, auf.

\section{Prävention}

Das BUK-NOG regelt nicht nur Organisationsfragen. Mit diesem Gesetz wird für die Prävention klargestellt, dass sich die Pflicht des Arbeitgebers, eine Gefährdungsbeurteilung durchzuführen, auch auf psychische Belastungen bei der Arbeit bezieht. Der Schutz der Gesundheit bei arbeitsbedingter psychischer Belastung ist auch ein Ziel der GDA (Gemeinsame Deutsche Arbeitsschutzstrategie) von Bund, Ländern und gesetzlicher Unfallversicherung.

Ein weiteres Ziel der GDA ist die Verringerung von arbeitsbedingten Gesundheitsgefährdungen und Erkrankungen im Muskel-Skelett-Bereich. Hierzu passt die Präventionskampagne der Unfallversicherung "Denk an mich. Dein Rücken“. Sie wurde im Januar 2013 erfolgreich gestartet und wird durch intensive Presseund Medienarbeit begleitet. Die Resonanz ist erfreulich groß.

\section{Qualität und Wirtschaftlichkeit}

Seit 2009 muss die DGUV (Deutsche Gesetzliche Unfallversicherung) jährlich dem Bundesministerium für Arbeit und Soziales über die Entwicklung der Verwaltungs- und Verfahrenskosten bei den gewerblichen Berufsgenossenschaften sowie die Maßnahmen zur Kostenoptimierung berichten. Nach dem letzten Bericht wurde vom Ministerium ausdrücklich die Erwartung geäußert, dass die Fusionen pauschal eine Rendite von $10 \%$ bei den Verwaltungs- und Verfahrenskosten erwirtschaften. Dem widersprach die Unfallversicherung und machte deutlich, dass sich solche pauschalen Ziele angesichts der Unterschiedlichkeit der Berufsgenossenschaften verbieten. Außerdem ist die Erstellung des Haushalts des einzelnen Trägers ein originäres Recht der Selbstverwaltung, welches mit Verantwortungsbewusstsein und Augenmaß ausgeübt wird. Wer die Selbstverwaltung lobt und wichtig findet, der muss auch ihre Rechte achten.

Der 18.05.2013 stellt ein erwähnenswertes Jubiläum dar; der Tag der Selbstverwaltung im Jahr 2013 markiert den 60. Jahrestag dieses wichtigen Prinzips in der Sozialversicherung: Vor 60 Jahren 
konnten Beitragszahler in Sozialwahlen erstmals darüber abstimmen, wer ihre Interessen in den Parlamenten der Sozialversicherungsträger vertreten soll. Dank dieser Selbstverwaltung sind Berufsgenossenschaften und Unfallkassen keine anonymen Behörden. Die Vertreter der Arbeitgeber und Versicherten prägen in entscheidender Weise unsere Arbeit. Sie bringen die Erfahrungen und Bedürfnisse von Millionen Beschäftigten, Arbeitgebern und Schülern in die Arbeit der gesetzlichen Unfallversicherung ein. Damit setzen sie Maßstäbe für unsere Arbeit in Prävention und Rehabilitation.

Eine Fusionsrendite kann nicht nur an einer Kostenreduktion gemessen werden; sie muss auch Qualitätsgewinne angemessen berücksichtigen. Wichtig ist eine vernünftige Relation zwischen Wirtschaftlichkeit und Qualität. Beides muss in einem angemessenen Verhältnis stehen. So ist z. B. bei der Neuausrichtung der Heilverfahren der erwartete Qualitätsschub Maßstab unseres Handelns. Die Unfallversicherung wird aber noch stärker als bisher transparent machen müssen, welche Kosten hinter ihren Dienstleistungen stehen und welche Wirkungen diese Dienstleistungen entfalten. Damit wird deutlich, dass die Unfallversicherung den Einsatz betriebswirtschaftlicher Steuerungsinstrumente forcieren muss.

\section{Benchmarkingprojekt Fallsteuerung}

Benchmarking ist schon heute fester Bestandteil der Steuerung bei den UV-Trägern - intern und auch trägerübergreifend. Für die Gemeinschaft aller Träger ist es ein Gewinn; so können sie die besten Problemlösungen finden und gute Erfahrungen von anderen übernehmen. Zudem schafft die gemeinsame Arbeit ein wertvolles Erfahrungsnetzwerk, auch über einzelne Projekte hinaus. Exemplarisch sei hier über das bisher größte Benchmarkingprojekt in der Unfallversicherung „Effektivität und Wirtschaftlichkeit der Fallsteuerung" berichtet. An diesem Projekt nahmen alle Berufsgenossenschaften und 14 Unfallkassen teil. Die unterschiedlichen Verfahrensweisen der Träger wurden untersucht in ihren Auswirkungen auf:
- die Qualität,

- die Bearbeitungszeiten,

- die Verwaltungskosten und

- die Leistungsausgaben.

In einem Prozessbenchmarking wurden Abläufe verglichen, Unterschiede identifiziert und letztlich konkrete Handlungsempfehlungen zur Optimierung der Prozesse abgeleitet. Einbezogen wurde die gesamte Bandbreite der Rehabilitation von der Bearbeitung leichter Unfälle bis hin zur intensiven Heilverfahrenssteuerung bei schwer Unfallverletzten. Daten wurden durch System- und Aktenauswertungen erhoben. In der qualifizierten Heilverfahrenssteuerung wurden anhand von Beispieldiagnosen vertiefende Analysen durchgeführt.

Das Projekt belegte deutlich, dass die Rehabilitation der gesetzlichen Unfallversicherung sehr erfolgreich ist. Bei den untersuchten Diagnosen mit erheblichen Verletzungen lag die Wiedereingliederungsquote in die gleiche oder eine ähnliche berufliche Tätigkeit bei beachtlichen $97 \%$. Das Projekt zeigte auch, dass es notwendig ist, Unfallverletzte individuell und möglichst frühzeitig zu betreuen - am besten schon in der Akutphase. Intensive Steuerung bedeutet für die Unfallversicherungsträger den persönlichen Kontakt vor Ort mit den Versicherten und eine konkrete Arbeitsplatzorientierung der Rehabilitation. Ein erwartetes, aber jetzt auch bewiesenes Ergebnis des Projekts ist, dass ein höherer Steuerungsgrad bei schweren Verletzungen tendenziell die Arbeitsunfähigkeit verkürzt und auch zu geringeren MdE-Folgen (MdE: Minderung der Erwerbsfähigkeit) führt. Das kann beispielhaft für die Diagnose Unterschenkelfraktur belegt werden: Die Fälle, die intensiv gesteuert werden, weisen eine deutlich niedrigere Dauer der Arbeitsunfähigkeit auf als die Fälle, die mit geringerer Intensität gesteuert werden. Der Unterschied beträgt im Durchschnitt 80 Tage. Auch für andere Beispieldiagnosen konnte ein positiver Zusammenhang zwischen Steuerungsgrad und Dauer der Arbeitsunfähigkeit festgestellt werden.

Im Fazit führt eine intensive Steuerung des Heilverfahrens zu besseren Rehabilitationsergebnissen, was an kürzerer
Arbeitsunfähigkeitsdauer, besseren Ausheilungsergebnissen und damit niedrigerer MdE und letztendlich auch geringeren Ausgaben evident wird. Dies steht folgendem Aufwand gegenüber: Im Schnitt werden pro Reha-Managementfall 40 bis 50 Bearbeitungsstunden erbracht. Das entspricht etwa $1500 €$ pro Fall. Setzt man demgegenüber pro vermiedenem Arbeitsunfähigkeitstag einen Wert von $90 €$ an, errechnen sich für 80 Arbeitsunfähigkeitstage $7200 €$, was den finanziellen Vorteil deutlich belegt. Außerdem werden aus Sicht des Versicherten die Krankheitsund damit die Leidensphase kürzer, und der Arbeitgeber profitiert von einer früheren Rückkehr seines unfallverletzten Mitarbeiters an den Arbeitsplatz - und dies bei geringeren Kosten beim Unfallversicherungsträger und damit einer Beitragsersparnis.

Eine konsequente, an den individuellen Bedürfnissen ausgerichtete intensive Betreuung von Versicherten erfordert engagierte und qualifizierte Mitarbeiter. Dies ist ein gutes Beispiel dafür, dass Personalkosten für qualifizierte Mitarbeiter die Leistungskosten positiv beeinflussen, also nicht zwangsläufig sozusagen schlechte Kosten sind - ein wichtiges Argument in der politischen Diskussion.

\section{Reha-Management}

Die oben angeführten Ergebnisse bestärkten die Berufsgenossenschaften und Unfallkassen darin, Unfallverletzte durch Reha-Manager intensiv zu unterstützen. Zur Identifizierung der intensiv betreuungsbedürftigen Fälle dienen eine prognostizierte Arbeitsunfähigkeitsdauer von mehr als 112 Tagen und/oder das Vorliegen von Komplikationen und relevante Kontextfaktoren. Solche Kontextfaktoren sind beispielsweise besondere Arbeitsplatzbedingungen, die familiäre Situation, Migrationshintergrund oder auch psychische Auffälligkeiten.

Im Zentrum des Reha-Managements stehen die individuelle, persönliche Beratung und Begleitung der Versicherten; sie setzen so früh wie möglich ein und beruhen auf einer partnerschaftlichen Einbindung aller Beteiligten.

Das Reha-Management dient dazu, alle notwendigen Maßnahmen schon vor 
Beginn der medizinischen Rehabilitation zu koordinieren. Frühzeitige Planung ermöglicht eine nahtlose Vernetzung von Maßnahmen, also von der Akutbehandlung über die medizinische Rehabilitation bis hin zur beruflichen und sozialen Wiedereingliederung. Deshalb sieht die Unfallversicherung in der Erstellung eines individuellen Reha-Plans, der von allen Beteiligten unterschrieben wird, ein wichtiges Instrument zur dauerhaften beruflichen und sozialen Wiedereingliederung.

Das Reha-Management entwickelte sich in den letzten Jahren gut. Es ist ein Alleinstellungsmerkmal der gesetzlichen Unfallversicherung und ein Aushängeschild erster Güte. Reha-Management wird auch in den Unfallversicherungssystemen der Schweiz und Österreichs betrieben. Die DGUV, die AUVA (Allgemeine Unfallversicherungsanstalt Österreichs) und die SUVA (Schweizerische Unfallversicherungsanstalt) initiierten ein gemeinsames Grundsatzpapier. Damit werden das gemeinsame Verständnis von Reha-Management dokumentiert und der Anspruch an eine effektive und betriebsnahe Fallsteuerung formuliert. Das Papier unterstreicht, dass sich das Reha-Management für die Versicherten und unter dem Gesichtspunkt der Effizienz lohnt. Die Broschüre wird mehrsprachig (in Deutsch, Englisch, Französisch und Italienisch) herausgegeben und stellt im In- und Ausland das im deutschsprachigen Raum praktizierte Reha-Management als gutes und nachahmenswertes Beispiel dar.

\section{Psychotherapeuten-Verfahren}

Seit der UMed (Unfallmedizinische Tagung) des Landesverbands Südwest der DGUV im Jahr 2011 wurde von der DGUV - neben den Aktivitäten zur Neuordnung der Heilverfahren - ein weiteres besonders praxisrelevantes Verfahren begonnen: Zum 01.07.2012 trat das neue Psychotherapeuten-Verfahren (PT-Verfahren) der gesetzlichen Unfallversicherung in Kraft. Es löst das bisherige Modellverfahren ab, das schon mehrfach Thema bei den UMed war. Die guten Erfahrungen mit diesem Modellverfahren führten jetzt zu einem bundeseinheitlichen Verfahren - unter Einbindung praktischer Erfahrun-

Trauma Berufskrankh 2014 · 16[Suppl 3]:219-222 DOI 10.1007/s10039-014-2072-8

(c) Springer-Verlag Berlin Heidelberg 2014

Th. Köhler

\section{Bericht zur sozialpolitischen Entwicklung}

\section{Zusammenfassung}

Fusionsprozesse. Das Gesetz zur Neuorganisation der bundesunmittelbaren Unfallkassen (BUK-NOG) schuf die rechtliche Grundlage für weitere Fusionen im öffentlichen Sektor der gesetzlichen Unfallversicherung. In der landwirtschaftlichen Sozialversicherung ist der Fusionsprozess jetzt abgeschlossen. Fusionsrendite/Kosten und Qualität. Die von Seiten der Politik von der Unfallversicherung erwartete Fusionsrendite muss Kostenund Qualitätsaspekte berücksichtigen. Der Nutzen einer intensiven Heilverfahrenssteuerung ist durch das bisher größte Benchmarkingprojekt in der gesetzlichen Unfallversicherung belegt. Reha-Management (Rehabilitationsmanagement) als individuelle Beratung und Begleitung der Versicherten ist in- zwischen ein gut etabliertes Alleinstellungsmerkmal der gesetzlichen Unfallversicherung in Deutschland, aber auch in Österreich und in der Schweiz. Mit dem Psychotherapeuten-Verfahren soll der Chronifizierung psychischer Störungen nach einem Arbeitsunfall oder einer Berufskrankheit adäquat und frühzeitig entgegengewirkt werden.

Ausblick. Für die neue Legislaturperiode zeichnen sich für die gesetzliche Unfallversicherung keine konkreten Gesetzesvorhaben $a b$.

\section{Schlüsselwörter}

Neuorganisationsgesetz - Fusionsrendite . Benchmarking · Reha-Management .

Psychotherapeuten-Verfahren

\section{Report on the sociopolitical development}

\section{Abstract}

Mergers. The law on the reorganization of the direct federal accident insurers (BUKNOG) provides the legal basis for further mergers within the statutory accident insurance sector. The merger process in the agricultural social insurance is now finished. Merger returns/costs and quality. The merger returns of accident insurers expected by politics must include aspects of costs and quality. The benefits of an intensive control of the healing process have been confirmed by the largest benchmarking project in statutory accident insurance. Rehabilitation management by means of an individualized consulting and accompanying of insured per- sons is now a well-established unique characteristic of the statutory insurance in Germany, Austria and Switzerland. The chronification of mental disorders after an occupational accident or disease can be adequately and timely counteracted by the psychotherapist procedure.

Perspective. No concrete changes are contemplated by the statutory accident insurance for the new legislative period.

\section{Keywords}

Reorganization law · Merger returns . Benchmarking $\cdot$ Rehabilitation . Psychotherapy gen der UV-Träger, insbesondere aus dem Reha-Management, sowie weiterer ärztlicher und psychologischer Kompetenz. Ziel ist es, Menschen, die durch einen schweren Arbeitsunfall oder eine Berufskrankheit an psychischen Störungen leiden, adäquat und rechtzeitig zu versorgen, damit die Beschwerden nicht chronifizieren.

Die Therapeuten im Netzwerk der Unfallversicherung müssen die Behandlung innerhalb 1 Woche übernehmen. Beibehalten wurde die schnelle Intervention, indem die Unfallversicherungsträger bis zu 5 probatorische Sitzungen unkompliziert bewilligen. Damit gibt es eine Ba- sis für eine gute Struktur- und Prozessqualität auf der Seite der Leistungserbringer. Der komplementäre Baustein auf Seiten der UV-Träger ist das Reha-Management, um auch bei psychischen Störungen für die betroffenen Versicherten eine erfolgreiche Rehabilitation erreichen $\mathrm{zu}$ können.

\section{Ausblick auf die Gesetzgebung}

In der Zeit einer geschäftsführenden Übergangsregierung und während laufender Koalitionsverhandlungen lässt sich nicht beurteilen, ob in der nächsten Legislaturperiode weitere Reformen auch 


\section{Eröffnung und Begrüßung}

für die Unfallversicherung anstehen. Bei der sich abzeichnenden Koalition scheint keine neue Privatisierungsdiskussion auf die gesetzliche Unfallversicherung zuzukommen.

Das Thema Wegeunfall wird von Arbeitgeberseite gelegentlich unter dem Blickwinkel der Unternehmerhaftpflicht in Frage gestellt. Für eine Herausnahme aus dem Versicherungsportfolio dürfte es aber auch jetzt an einer politischen Mehrheit fehlen.

$\mathrm{Ob}$ die im letzten Anlauf vor wenigen Jahren gescheiterte Reform des Leistungsrechts -zu nennen ist hier die Rententhematik - zeitnah wiederaufgegriffen werden wird, ist nicht abzusehen. Denkbar ist, dass die Beweisprobleme im Berufskrankheitenverfahren und damit verbundene Reformvorstellungen die Politik und damit auch die Unfallversicherungsträger erneut beschäftigen werden. Interessant wird auch sein, ob und in welcher Form das in letzter Minute im Bundesrat gescheiterte Präventionsgesetz doch noch realisiert wird und inwieweit die Unfallversicherung davon betroffen ist.

\section{Korrespondenzadresse}

\section{Th. Köhler}

Deutsche Gesetzliche Unfallversicherung

(DGUV), Landesverband Südwest,

69004 Heidelberg

thomas.koehler@bgrci.de

\section{Einhaltung ethischer Richtlinien}

Interessenkonflikt. Th. Köhler gibt an, dass kein Interessenkonflikt besteht.

Dieser Beitrag beinhaltet keine Studien an Menschen oder Tieren.

The supplement containing this article is not sponsored by industry. 